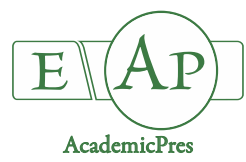

\title{
Plant Root Hair Growth in Response to Hormones
}

\author{
Dejian ZHANG ${ }^{1,2}$, Chunyan LIU ${ }^{1,2}$, Yujie YANG ${ }^{1,2}$, \\ Qiangsheng WU ${ }^{1,2,3 *}$, Yeyun $\mathrm{LI}^{2 * *}$ \\ ${ }^{1}$ Yangtze University, Hubei Key Laboratory of Waterlogging Disaster and Agricultural Use of Wetland, Jingzhou, Hubei 434020, P.R. \\ China; zhangdejian0553@126.com; chunyanliu_2009@126.com;yjyang@yangtzeu.edu.cn;wuqiangsh@163.com(*correspondingauthor) \\ ${ }^{2}$ Anhui Agricultural University, State Key Laboratory of Tea Plant Biology and Utilization, \\ 130 Changjiang West Road, Hefei, 230036, Anhui, P.R. China; lyy@ahau.edu.cn ( ${ }^{* *}$ corresponding author) \\ ${ }^{3}$ University of Hradec Kralove, Faculty of Science, Department of Chemistry, Hradec Kralove 50003, Czech Republic
}

\begin{abstract}
Plant root hair is tubular projections from the root epidermis. Its can increase root surface area, which is very important for nutrients and water uptake as well as interaction with soil microorganisms. In this short review, we discussed the effects of hormones (auxin, ethylene, jasmonic acid, methyl jasmonate, strigolactones, and brassinosteroids) on root hair growth. It was highlight the interaction between auxin and ethylene on root hair growth. Furthermore, the mechanisms of jasmonic acid, methyl jasmonate, strigolactone and brassinosteroids on root hair growth may through auxin or ethylene signaling pathway partly. In future, more genes relating to root hair growth needed clone and elucidate their roles, as well as undertaking reverse genetics and mutant complementation studies to add the current knowledge of the signaling networks, which are involved in root hair growth that regulated by hormones.
\end{abstract}

Keywords: auxin; brassinosteroids; ethylene; jasmonic acid; methyl jasmonate; root hair; strigolactones

\section{Introduction of root hair}

Root hairs can increase root surface area greatly and enhance the absorption of nutrients and water (Cao et al., 2013; Vincent et al., 2017; Dolan, 2017; Liu et al., 2018 a,b). Its growth always consists of four stages, viz., cell fate specification, initiation, tip growth and maturation (Fig. 1).

In the stage of cell fate specification, TRYPTICHON $(T R Y)$ and CAPRICE (CPC) genes were been confirmed specific expression in the trichoblasts (epidermis which bulge to root hair), that positively regulated root hair formation (Wada et al., 1997; Schellmann et al., 2002; Savage et al., 2008; Libault et al., 2010). In contrast, GLABRA2 (GL2), TRANSPARENT TESTA GLABRA $(T T G)$, and $W E R E W O L F(W E R)$ were specific expression in the atrichoblasts (epidermis which cannot initiate to root hair), that negatively regulated root hair initiation (Cristina et al., 1996; Masucci et al., 1996; Walker et al., 1999; Zhu et al., 2017). The initiation patterns of root hair have been divided into 3 types: asymmetrical cell division type, random type and positionally cued type (Clowes, 2000; Kim et al., 2006a). In asymmetrical cell division type, the root epidermis (such as Oryza sativa) consists of two sizes of cells, long and short, but only the short cell can differentiates into root hair cell (Kim and Dolan, 2011). In random type, root hair cell can differentiate from any root epidermis of plants, such as citrus (Zhang et al., 2013) and Soleirolia soleirolii (Clowes, 2000). The positional cued type has been found in Arabidopsis: a trichoblast is located over a single cortical cell, whereas trichoblast overlying the junction of two cortical cells (Clowes, 2000; Dolan and Costa, 2001). In root hair tip growth, the deposition of new plasma membrane and cell wall material are confined to the expanding tip and the cytoplasm of the hair are highly polarized, with secretory vesicles concentrated located behind the hair tip, followed by the organelles required for the production and secretion of new cell wall and plasma membrane materials (Galway et al., 1997; Carol and Dolan, 2002, 2006; Nielsen, 2009). During root hair maturation, ribosomes, mitochondria, and endoplasmic reticulum concentrate at the root hair tip (Nestler et al., 2014).

Root hair growth could be influenced by various factors such as soil edaphon, culture substrates, plant growth regulators, mineral nutrients and so on. However, many researchers focused on the effects of auxin, ethylene, jasmonic acid, methyl jasmonate, strigolactone, brassinosteroids on plant root hair development and growth. 


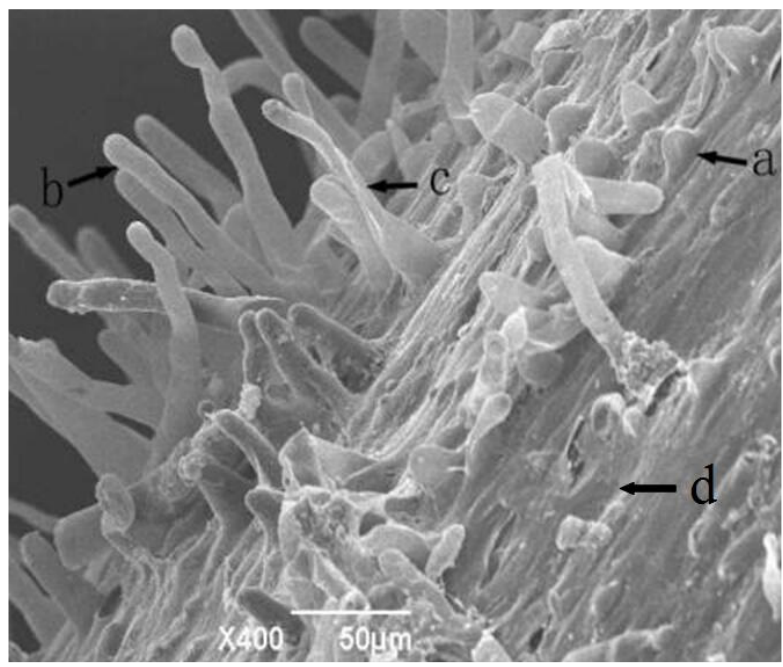

Fig. 1. The growth stages of root hair of citrus. Note: ainitiation, b-tip growth, c-maturation, d-cell fate specification

\section{Root hair growth in response to hormones}

Auxin

Auxin is considered as the important signaling molecule involved in regulating root hair growth (Liu et al., 2018a, b). Exogenous auxins (1-naphthylacetic acid and Indole butyric) can promote root hair growth significantly (Rahman et al., 2002; Liu et al., 2016; Zhang et al., 2018). In Arabidopsis, the auxin response mutant axrl and auxl have shorter and fewer root hairs for missing auxin signal (Rahman et al., 2001, 2002). Based on transcriptome sequencing data, $90 \%$ of genes related to root hair growth were positively regulated by auxin (Bruex et al., 2012).

It has been demonstrated that PIN, ATP-BINGING CASSETTE B (ABCB), AUXIN RESISTANT 1 (AUXI) and LIKE AUXI (LAX) genes which regulating auxin transport in root has positive correlation with root hair growth (Rahman et al., 2002; Ganguly et al., 2010; Zhang et al., 2018). 1-naphthoxyacetic acid and 2-naphthoxyacetic acid, as auxin transport inhibitors, blocked root hair growth for missing auxin signal (Rahman et al., 2002; Zhang et al., 2018). The auxin transportation channel from root tip to root hair zone was been blocked in Arabidopsis mutant ein2, which blocked root hair growth (Rahman et al., 2002). Auxin synthesis which controlled by tryptophan aminotransferase related (TARs), flavin-containing monooxygenase (YUCs), etc, also affects root hair growth (Zhang et al., 2016, 2018). Understanding auxin biosynthesis is another factor in understanding root hair growth.

\section{Ethylene}

Ethylene is considered as another vital signal molecule involved in regulating root hair growth (Pitts et al., 1998). Zhang et al. (2016) confirmed that ethylene stimulate the growth of root hair of citrus. EIN2 as a positive regulator of ethylene responses, has been demonstrated that inducing root hair growth (Rahman et al., 2002; Zheng and Zhu
2016). In Arabidopsis, the ethylene response mutations ein2 and etrl have fewer root hairs, while the ethylene overproducing mutant etol has longer root hairs (Masucci and Schiefelbein, 1996; Pitts et al., 1998; Rahman et al., 2002). Furthermore, the ethylene precursor (1aminocyclopropane-1-carboxylic acid) could induce root hair growth, whereas ethylene biosynthesis inhibitors (aminoethoxyvinylglycine and $\mathrm{AgNO}_{3}$ ) can block it (Leblanc et al., 2008; Zhang et al., 2016).

What's fascinating is that the interaction between ethylene and auxin on root hair growth. The ethylene precursor (1-aminocyclopropane-1-carboxylic acid) could restore root hair growth in Arabidopsis auxin response mutant axrl, while exogenous auxin (naphthylacetic acid) can relieve the inhibitory effect on root hair growth in ethylene signal transduction mutant ein2 (Pitts et al., 1998; Rahman et al., 2002; Muday et al., 2012). On one side, endogenous auxin plays a major role in root hair growth, and its concentration in trichoblast determines the initiation and growth of root hair (Jones et al., 2009; Ganguly et al., 2010). On the other side, ethylene is a key signal of root hair growth for that of growth regulators or mineral nutrients regulate root hair growth by control the concentration of endogenous ethylene in root hair cells (Michael et al., 2001). Ethylene could affect endogenous auxin level by regulating its biosynthesis and transport way, which is important for root and root hair initiation (Rahman et al., 2002; Růžička et al., 2007; Zhang et al., 2016). Furthermore, Auxin can stimulate ethylene biosynthesis, which regulating root hair growth positively (Pitts et al., 1998; Muday et al., 2012).

\section{Jasmonic acid and Methyljasmonate (JA and MeJA)}

$\mathrm{JA}$ and MeJA can also regulate root hair growth positively (Zhu et al., 2006; Liu et al., 2016). However, there has interaction between JA/MeJA and ethylene on regulating root hair growth. On the one side, the positive effects of JA and MeJA on root hair growth can diminish in ethylene inhibitor (aminoethoxyvinylglycine or $\mathrm{AgNO}_{3}$ ) treatment and in Arabidopsis ethylene-insensitive mutant etrl (Zhu et al., 2006). On the other side, the JA biosynthesis inhibitor (ibuprofen or salicylhydroxamic acid) not only diminished the facilitating effect of ethylene precursor (1-aminocyclopropane-1-carboxylic acid) on root hair growth, but also decreased the growth of root hair in ethylene over-producing mutant etol (Zhu et al., 2006).

\section{Strigolactone (SLs)}

Recently studies shown that SLs are a novel class of plant hormones that regulate plant's shoot and root growth (Xie et al., 2010; Waters et al., 2017). GR24 (a synthetic bioactive SL) positively regulated root hair growth in Arabidopsis (Kapulnik et al., 2011). However, excess GR24 could lead to disrupting auxin transport and high level of auxin in root hair, which has a negative effect on root hair growth (Kapulnik et al., 2011). Studies have presented that SLs affect root hair growth by the genes of PINs and TIRI which regulating auxin transport in root (Koltai et al., 2010; Kapulnik et al., 2011; Mayzlish-Gati et al., 2012). Hence, there has interaction between SLs and auxin on regulating root hair growth. 
280

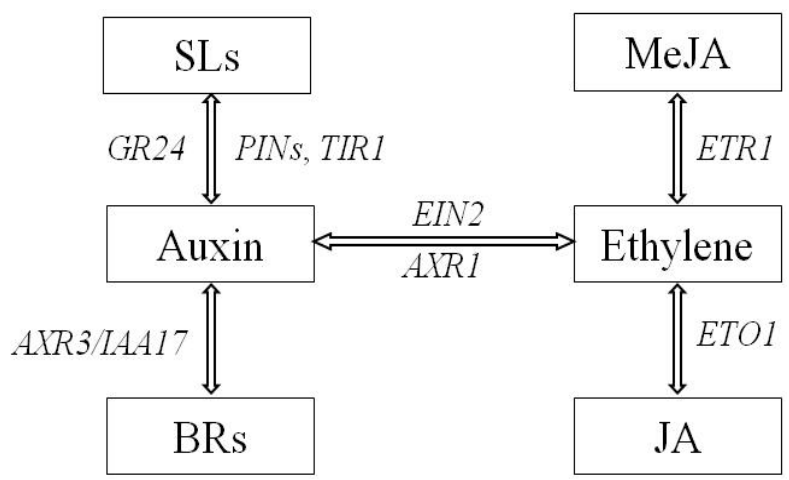

Fig. 2. The model of the interactions among hormones in regulating root hair growth

\section{Brassinosteroids (BRs)}

BRs have different effects on root hair growth, that it reduced the growth of root hairs in Arabidopsis but induced its growth in Oryza sativa (Kim et al., 2006b; Hardtke et al., 2007). Studies showed that $A X R 3 / L A A 17$ involving in $\mathrm{BR}$ signaling pathway (Mouchel et al., 2004, 2006; Kim et al., 2006b). In addition, the gain-of-function mutations axr3/iaal 7 inhibited root hair growth by affecting $B R$ signal (Knox et al., 2003). Thus, there has a cross-talk of BR and auxin in regulating root hair growth.

\section{Future perspectives and Conclusions}

There have complicated interactions among hormones in regulating root hair growth (Fig. 2). However, auxin and ethylene might play leading roles in root hair growth. Improving plants to make root hair high efficiency of water and nutrients uptake should increase crop production. Even abundant research papers carry on deeper studying on root hair growth, but root hair morphogenesis is driven by an amalgam of interacting processes controlled by complex signaling events, such as auxin, ethylene, etc. It is not clear how these signaling components interaction regulating root hair at the molecular and cellular level. More works are need to clone the genes of additional root hair mutants and undertake reverse genetics and mutant complementation studies to add to our current knowledge of the signaling networks involving hormones regulating root hair growth.

\section{Acknowledgements}

This work was supported by the Hubei Key Laboratory of Waterlogging Disaster and Agricultural Use of Wetland (grant number KF201807), the Open Fund of State Key Laboratory of Tea Plant Biology and Utilization (grant number SKLTOF20180113) and Young and Middle-aged Talent Project of Hubei Provincial Education Department (grant number Q20181304).

\section{References}

Bruex A, Kainkaryam RM, Wieckowski Y, Kang YH, Bernhardt C, Xia Y, ... Schiefelbein J (2012). A gene regulatory network for root epidermis cell differentiation in Arabidopsis. PLoS Genetics 8:e1002446.
Cao X, Chen CL, Zhang DJ, Shu B, Xiao J, Xia RX (2013). Influence of nutrient deficiency on root architecture and root hair morphology of trifoliata orange (Poncirus trifoliata L. Raf.) seedlings under sand culture. ScientiaHorticulturae 162:100-105.

Carol RJ, Dolan L (2002). Building a hair: tip growth in Arabidopsis thaliana root hairs. Philosophical Transactions Biological Sciences 357:815-821.

Carol RJ, Dolan L (2006). The role of reactive oxygen species in cell growth: lessons from root hairs. Journal of Experimental Botany 57:1829-1834.

Clowes FAL (2000). Pattern in root meristem development in angiosperms. New Phytologist 146:83-94.

Cristina MD, Sessa G,Dolan L, Linstead P, BaimaS, RubertiI,MorelliG(1996). The Arabidopsis ATHB10 (GLABRA2) is a HD-ZIP protein required for repression of roothair development. Plant Journal 10:393-402.

Dolan L (2017). Root hair development in grasses and cereals (Poaceae). Current Opinion in Genetics Development 45:76-81.

Dolan L, Costa S (2001). Evolution and genetics of root hair stripes in the rootepidermis. Journal of Experimental Botany 52 (Suppl. 1):413-417.

Galway ME, Heckman JWJ, Schiefelbein JW (1997). Growth and ultrastructure of Arabidopsis root hairs: the $r h d 3$ mutation alters vacuole enlargement and tip growth. Planta 201:209-218.

Ganguly A, LeeSH, Cho M, LeeOR, Yoo H,ChoHT (2010). Differential auxin-transporting activities of PIN-FORMED proteins in Arabidopsis roothair cells. Plant Physiology 153:1046-1061.

Hardtke CS, Dorcey E, Osmont KS, Sibout R (2007). Phytohormone collaboration: zooming in on auxin-brassinosteroid interactions. Trends in Cell Biology 17:485-492.

Jones AR, Kramer EM, Knox K, Swarup R, Bennett MJ, Lazarus CM, ... Grierson CS (2009). Auxin transport through non-hair cells sustains root hair development. Nature Cell Biology 11:78-84.

Kapulnik Y, Delaux PM, Resnick N, Mayzlish-Gati E, Wininger S, Bhattacharya C, ... Koltai H (2011). Strigolactones affect lateral root formation and root hair elongation in Arabidopsis. Planta 233:209-216.

Kim DW, Lee SH, Choi SB, Won SK, Heo YK, Cho M, ... Cho HT (2006a). Functional conservation of a root hair cell-specific cis-element in angiosperms with different root hair distribution patterns. Plant Cell 18:2958-2970.

Kim H, Park PJ, Hwang HJ, Lee SY, Oh MH, Kim SG (2006b). Brassinosteroid signals control expression of the AXR3/LAAI7 gene in the cross-talk point with auxin in root development. Bioscience Biotechnology and Biochemistry 70:768-773.

Kim CM, Dolan L (2011). Root hair development involves asymmetric cell division in Brachypodium distachyon and symmetric division in Oryza sativa. New Phytologist 192:601-610.

Knox K, Grierson CS, Leyser O (2003). AXR3 and SHY2 interact to regulate roothair development. Development 130:5769-5777.

Koltai H, Dor E, Hershenhorn J, Joel DM, Weininger S, Lekalla S, ... Kapulnik Y (2010). Strigolactones' effect on root growth and root hair elongation may be mediated by Auxin-efflux carriers. Journal of Plant Growth Regulation 29:129-136.

Leblanc A, Renault H, Lecourt J, Etienne P, Deleu C, DeunffEL (2008). Elongation changes of exploratory and root hair systems induced by aminocyclopropane carboxylic acid and aminoethoxyvinylglycine affect nitrate uptake and $B n N r t 2.1$ and $B n N r t 1.1$ transporter gene expression in oilseed rape. Plant Signaling\& Behavior 146(4):1928-1940. 
Libault M, Brechenmacher L, Cheng J, Xu D, Stacey G (2010). Root hair systems biology. Trends Plant Science 15:641-650.

Liu CY, Srivastava AK, Zhang DJ, Zou YN, Wu QS (2016). Exogenous phytohormones and mycorrhizas modulate root hair configuration in trifoliate orange. Notulae Botanicae Horti Agrobotanici Cluj-Napoca $44(2): 548-556$.

Liu CY, Wang P, Zhang DJ, Zou YN, Kuča K, Wu QS (2018a). Mycorrhiza-induced change in root hair growth is associated with IAA accumulation and expression of EXPs, in trifoliate orange under two $\mathrm{P}$ levels. Scientia Horticulturae 234:227-235.

Liu CY, Zhang F, Zhang DJ, Srivastava AK, Wu QS, Zou YN (2018b). Mycorrhiza stimulates root hair growth and IAA synthesis and transport in trifoliate orange under drought stress. Scientific Reports 8:1978.

MasucciJD, Rerie WG, Foreman DR, Zhang M, Galway ME, Marks MD, Schiefelbein JW (1996). The homeobox gene GLABRA2 is required for position-dependent cell differentiation in the root epidermis in Arabidopsis thaliana. Development 122:1253-1260.

Masucci JD, Schiefelbein JW (1996). Hormones act downstream of TTG and GL2 to promote root hair outgrowth during epidermis development in the Arabidopsis root. Plant Cell 8:1505-1517.

Mayzlish-GatiE, De-Cuyper C, GoormachtigS, Beeckman T, Vuylsteke M, Brewer PB, ... Koltai H (2012). Strigolactones are involved in root response to low phosphate conditions in Arabidopsis. Plant Physiology 160:1329-1341.

Michael G (2001). The control of root hair formation: suggested mechanisms. Journal of Plant Nutrition and Soil Science 164:111-119.

Mouchel CF, Briggs GC, Hardtke CS (2004). Natural genetic variation in Arabidopsis identifies BREVIS RADIX, a novel regulator of cell proliferation and elongation in the root. Genes Development 18:700714.

Mouchel CF, Osmont KS, Hardtke CS (2006). BRX mediates feedback between brassinosteroid levels and auxin signalling in root growth. Nature 443:458-461.

Muday GK, Rahman A, Binder BM (2012). Auxin and ethylene: collaborators or competitors. Trends in Plant Science 17:181-195.

Nestler J, Liu S, Wen TJ, Paschold A, Marcon C, Tang HM, ... Hochholdinger $\mathrm{F}$ (2014). Roothairless5, which functions in maize (Zea mays $\mathrm{L}$.) root hair initiation and elongation encodes a monocot-specific NADPH oxidase. Plant Journal 79:729-740.

Nielsen E (2009). Plant cell wall biogenesis during tip growth in root hair cells. Root Hairs 12:85-102.

Pitts RJ, Cernac A, Estelle M (1998). Auxin and ethylene promote root hair elongation in Arabidopsis. Plant Journal 16:553-560.

Rahman A, Amakawa T, Goto N, Tsurumi S (2001). Auxin is a positive regulator for ethylene-mediated response in the growth of Arabidopsis roots. Plant and Cell Physiology 42:301-307.

Rahman A, Hosokawa S, Oono Y, Amakawa T, Goto N, Tsurumi S (2002). Auxin and ethylene response interactions during Arabidopsis root hair development dissected by auxin influx modulators. Plant Physiology 130:1908-1917.
Rưžička R, Ljung K, Vanneste S, Podhorská R, Beeckman T, Friml J, Benková $E$ (2007). Ethylene regulates root growth through effects on auxin biosynthesisand transport-dependent auxin distribution. Plant Cell 19:2197-2212.

Savage NS, Walker T, Wieckowski Y, Schiefelbein J, Dolan L, Monk NA (2008). A mutual support mechanism through intercellular movement of CAPRICE and GLABRA3 can pattern the Arabidopsis root epidermis. PLoSBiology 6:e235.

Schellmann S, Schnittger A, Kirik V, Wada T, Okada K, Beermann A, ... Hülskamp M(2002). TRIPTYCHON and CAPRICE mediate lateral inhibition during trichome and root hair patterning in Arabidopsis. EMBO Journal 21:5036-5046.

Vincent C, Rowland D, Na C, Schaffer B (2017). A high-throughput method to quantify root hair area in digital images taken in situ. Plant and Soil 412:61-80.

Wada T, Tachibana T, Shimura Y, Okada K (1997). Epidermal cell differentiation in Arabidopsis is determined by a Myb homolog, CPC. Science 227:1113-1116.

Walker AR, Davison PA, Bolognesi-Winfield AC, James CM, Srinivasan N, Blundell TL, ... Gray JC (1999). The TRANSP ARENT TESTA GLABRAI locus, which regulates trichome differentiation and anthocyanin biosynthesis in Arabidopsis, encodes a WD40 repeat protein. Plant Cell 11(7):1337-1350.

Waters MT, Gutjahr C, Bennett T, Nelson DC (2017). Strigolactone signaling and evolution. Annu. Rev. Plant Biology 68:291-322.

Xie X, Yoneyama K, Yoneyama K (2010). The strigolactone story. Annual Review of Phytopathology 48:93-117.

ZhangDJ,Xia RX, CaoX,ShuB, Chen CL (2013). Roothair development of Poncirus trifoliata grown in different growth cultures and treated with 3-indolebutyric acid and ethephon. Scientia Horticulturae 160:389397.

Zhang DJ, Xia RX, Cao X (2016). Ethylene modulates root hair development in trifoliate orange through auxin-signaling pathway. Scientia Horticulturae 213:252-259.

Zhang DJ, Yang YJ, Liu CY, Zhang F, Hu W, Gong SB, Wu QS (2018). Auxin modulates root hair growth through its signaling pathway in citrus. Scientia Horticulturae 236:73-78.

Zheng Y, Zhu Z (2016). Relaying the ethylene signal: new roles for EIN2. Trends in PlantScience 21:2-4.

Zhu C, Gan L, Shen Z, Xia K (2006). Interactions between jasmonates and ethylene in the regulation of root hair development in Arabidopsis. Journal of Experimental Botany 57:1299-1308.

Zhu Y, RongL, Luo Q, Wang B, Zhou N, Yang Y, ... Dong A (2017). The histone chaperone NRP1 interacts with WEREWOLF to activate GLABRA2 in Arabidopsis root hair development. Plant Cell 29:260276. 